\title{
Seek and treat to optimize HIV and AIDS prevention
}

\author{
Réka Gustafson MD, Julio Montaner MD, Barbara Sibbald BJ
}

$\mathrm{S}$ ince 1996, highly active antiretroviral therapy (HAART) has transformed HIV into a chronic, manageable condition. HAART fully suppresses HIV replication, driving the HIV load in plasma to undetectable levels. This allows for immune reconstitution, long-term remission and dramatic reductions in morbidity and death. Today, a 20-year-old who receives a diagnosis of HIV and treatment with HAART can expect to live until the age of 73 years.

In addition to benefiting individual patients, HAART has been shown to prevent vertical and parenteral transmission of HIV. ${ }^{2}$ More recently, a prospective randomized trial showed that use of HAART decreased HIV transmission among HIVserodiscordant couples by more than $95 \% .^{3}$ The populationlevel preventive impact of HAART has led the US government to announce a policy goal of "an AIDS-free generation."

HAART is widely available in Canada. However, we are not fully benefiting from it, because too many of those infected are diagnosed late, sometimes very late. The Public Health Agency of Canada estimates that at any one time at least a quarter of those infected are unaware of their infection, and more than $50 \%$ receive the diagnosis after immunodeficiency is established. ${ }^{4}$ Late diagnosis not only limits the effectiveness of HAART, it also promotes HIV transmission, because individuals who haven't received a diagnosis are less likely to take appropriate precautions.

HIV meets the widely accepted principles for screening programs. Its treatment is accepted, accessible and highly costeffective; its screening test is sensitive, specific and minimally invasive; and its early treatment has been well established as beneficial. But Canada has no recommendation for routine testing outside of prenatal screening and screening of the blood supply. We approach HIV testing as we did during the first decade of the pandemic. We focus on testing people whom we know to be at risk for HIV, despite good evidence that riskbased strategies miss a substantial proportion of cases.

Fortunately, there is opportunity for change. People who receive diagnoses late in their infection typically have had multiple encounters with the health care system before the diagnosis. In a Vancouver Coastal Health pilot project that set out to improve early diagnosis of HIV and care for people with the infection, researchers found that $60 \%$ of people diagnosed with a CD4 count of less than 350 cells $/ \mathrm{mm}^{3}$ had at least 1 laboratory test, emergency department visit or hospital admission in the previous 3 years or since their last negative test (unpublished data, 2010-2012). According to 2006 recommendations in the United States, ${ }^{6}$ and more recently in the United Kingdom ${ }^{7}$ and France, ${ }^{8}$ these patients should have been offered an HIV test at these earlier encounters, without needing to raise the subject or acknowledge a specific risk. The US recommends routine screening in all health care settings for patients aged 13 to 64 years. ${ }^{6}$ The UK's National Institute for Health and Clinical Excellence asks health professionals to consider offering and recommending an HIV test when registering and admitting new patients, ${ }^{7}$ and France recommends screening of the whole population aged 15 to 70 years and targeted screening of at-risk populations. ${ }^{8}$

As part of the Vancouver Coastal Health pilot project, administrators of acute care hospitals in Vancouver recommended HIV testing as part of all medical admissions and emergency department visits (unpublished data, 2010-2012). Although clinicians' offer of the test was suboptimal (at 43\%), patient acceptance was very high (at 92\%) and diagnostic yield was 6 new positives per 1000 HIV tests. Importantly, routine HIV testing is deemed cost-effective when the return is at least 1 HIV positive per 1000 tests. ${ }^{9}$ Thus, the medical health officer for Vancouver Coastal Health is now recommending routine HIV screening in acute and primary care in Vancouver.

Some questions remain to be answered. In particular, the best clinical opportunities for routine testing and the optimal frequency of testing of different age groups need to be defined through careful monitoring and evaluation of testing programs. But, as with all screening programs, the program must first be established, and then evaluated and modified as needed.

Other challenges to screening include the persistent stigma and discrimination toward HIV-infected people and those at risk. For example, HIV-infected people may face criminal prosecution following unprotected sex, even if HIV transmission does not occur, which can discourage some people from testing and therefore threatens to undermine public health efforts.

A proactively deployed "seek and treat" strategy will dramatically reduce AIDS-related morbidity and death, as well as HIV transmission, and as such provide the road map for an AIDS-free generation. ${ }^{10}$ We must re-examine our perceptions and assumptions, including the sole reliance on risk-based HIV testing. It is now evident that HIV testing, based only on perceived risk, misses multiple opportunities for earlier diagnosis. Routine HIV testing, on the other hand, has been shown to be acceptable and highly cost-effective. It is therefore imperative to implement and evaluate routine HIV testing across Canada.

For references, see Appendix 1, available at www.cmaj.ca/lookup/suppl/doi: 10.1503/cmaj.121810/-/DC1.

\section{Competing interests: None declared.}

Affiliations: Réka Gustafson is Medical Health Officer and Medical Director of Communicable Disease Control, Vancouver Coastal Health, Vancouver, BC. Julio Montaner is Director, BC Centre for Excellence in HIV/AIDS, Vancouver, BC. Barbara Sibbald is Deputy Editor, Analysis and Humanities, CMAJ.

Correspondence to: $C M A J$ editor, pubs@cmaj.ca

CMAJ 2012. DOI:10.1503/cmaj.121810 